\title{
SOME OBSERVATIONS ON CONTINUUM MECHANICS WITH EMPHASIS ON ELASTICITY
}

\section{J. J. STOKER}

1. Introductory remarks. Twenty years ago, I gave an invited address at a meeting of the Mathematical Society in New York. At that time also my subject was in the field of elasticity, and though I have worked perhaps more in other fields in the intervening years, I have never lost my interest in the subject and, particularly in the last few years, that interest has been strongly re-awakened. Consequently, I thought it reasonable to emphasize certain types of problems in elasticity in this lecture.

The subject of elasticity belongs, of course, to the general field of mechanics of continuous media. This is a classical field lying at the boundary between mathematics and physics which has played a very considerable role in the history of these two disciplines, with enrichments for both resulting through the fruitful interplay of the two. Some glimpses and reminders of what I mean will come through to you, I hope, in the course of my remarks today.

The field of continuum mechanics is a very large field, comprising, as it does, such diverse subjects as hydrodynamics, gas dynamics, elasticity, plasticity, electromagnetic theory, and one of the most recent and flourishing additions to the field, magneto-hydrodynamics. Even the field of elasticity is a very large field. Consequently, in order to be able to say anything intelligible in a one-hour lecture, it is necessary to restrict the subject matter. I have therefore decided to confine my remarks mainly to a small though important class of problems in the field, namely problems concerning the stability of equilibrium positions of elastic solids under stress. However, it is a fact that a large part, and an important part, of the theory of continuum mechanics can be developed in quite general terms and carried very far without being too specific about the nature of the medium to be treated.

It is feasible therefore to put my discussion in a proper mathematical context by giving first a brief outline of the general theory of mechanics of continuous media. This is followed by a discussion of the stability under compression of thin elastic solids, then of thick elastic solids. A return to bodies of zero thickness, i.e. elastic surfaces, is then made.

The thirty-fourth Josiah Willard Gibbs Lecture, delivered at Washington, D. C., on January 24, 1961, under the auspices of the American Mathematical Society; received by the editors November 28, 1961. 
Finally, I conclude with some observations of a general nature about mathematics in the United States, especially with respect to mathematics in its relation to the physical world.

2. The mechanics of continuous media. I consider a body in threedimensional space and define first of all the kind of deformations which are to be considered. By a deformation we mean that the points $P$ of the body given originally by a vector $\mathrm{x}=\left(x_{1}, x_{2}, x_{3}\right)$ go into the deformed position $\bar{P}$ given by a vector $\overline{\mathrm{x}}=\left(\bar{x}_{1}, \bar{x}_{2}, \bar{x}_{3}\right)$. The components of $\bar{x}$ are functions of the coordinates of the points in their original position and of the time $t$, that is, $\overline{\mathbf{x}}=f(\mathrm{x}, t)$, in which $f$ is vector valued, of course. It is assumed in continuum mechanics that the mapping so induced is a topological mapping and, in addition, that it has continuous derivatives of a certain order with respect to the space variables and the time. This assumption insures, among other things, that the boundary of the solid goes into the boundary of the deformed solid, that curves and surfaces remain curves and surfaces after a deformation, etc. The domain of the space variables $x_{i}$ is that occupied by the medium at the time $t=0$, and $t$ ranges over positive values $t \geqq 0$. Thus we operate with what are called (with dubious historical accuracy, it seems) the Lagrange variables.

Continuum mechanics is in a certain sense a branch of differential geometry in the large, which is, however, made more complicated by the necessity to consider the action of forces on the system of points under consideration. As in differential geometry in the large, one begins with local considerations. This is, in a way, forced upon one inevitably because the basic laws from which one starts from the point of view of mechanics are Newton's laws which, as everyone knows, are formulated in the first instance for mass particles. In continuum mechanics one assumes these laws to hold in the limit, when a particle is shrunk down on a point, and this includes, by the way, the third law of Newton, namely that actions and reactions of contiguous particles on one another are equal and opposite.

I turn then to considerations in the small, beginning with the notion of strain. This is a purely kinematic entity which can be completely characterized by the Jacobian matrix $p=p_{i k}$ of the transformation which carries the points $P$ into their images $\bar{P}$ in the deformed position. Thus we have

$$
p=p_{i k}=\left(\frac{\partial \bar{x}_{i}}{\partial x_{k}}\right),
$$

and the determinant $|p|$ of the matrix is assumed to be positive. The differential $d \bar{x}$ is then, of course, given by 


$$
d \bar{x}=p d x,
$$

with $d x$ and $d \bar{x}$ regarded as $1 \times 3$ matrices. (We shall use from now on a matrix notation, for the most part.) It is rather clear intuitively that the description of the strain condition near a given point requires, at the least, the knowledge of the manner in which the line elements $d s$ at that point are transformed into their images $d \bar{s}$ in the course of the deformation. We introduce therefore the relative elongations $e$ given by

$$
e=\frac{d \bar{s}-d s}{d s}=\left(\frac{d x^{*} p^{*} p d x}{d x^{*} d x}\right)^{1 / 2}-1 .
$$

The star refers to the transpose of a matrix. As we see it is the matrix $p^{*} p$ which serves to determine the elongations at a point when the line element varies in the directions fixed by $d x$. It is convenient to introduce a strain matrix $\eta$ by the formula

$$
\eta=\left(p^{*} p\right)^{1 / 2}-1 .
$$

This matrix exists since the square root of $p^{*} p$ can be taken because $p^{*} p$ is symmetric and positive. Upon comparison with (2.3) it is seen that the stationary values of $e$ (when $d x$ is varied) are the eigenvalues of $\eta$. These elongations are called the principal elongations.

In the greater part of the literature it is customary to take as a measure of the strain not the actual differences of length of line elements, but rather the differences of their squares, and thus to take as the strain matrix the following expression:

$$
\eta=\frac{1}{2}\left(p^{*} p-1\right) .
$$

The factor $1 / 2$ in this formula has as a result that for small strains, both (2.4) and (2.4)' lead to identical formulations. The great bulk of the literature deals with the case of small strains and hence it is then a matter of indifference which definition for $\eta$ is chosen. In spite of the occurrence of the square root in (2.4), this definition of the strain matrix is sometimes more convenient than that given by $(2.4)^{\prime}$.

I turn next to the notion of stress at a point. This notion was introduced in its full generality by Cauchy. One considers an element of area $d S$ with a definitely defined unit normal with components $\xi_{i}$ in the original undeformed position and their images $d \bar{S}$ and $\bar{\xi}_{i}$ in the deformed position. In the deformed position it is assumed that a force 
is exerted across the surface area due to the action of the material on one side of that surface on the material on the other side. The total force across the element of area $d \bar{S}$ can be expressed by the formula

$$
\sigma_{i k} \bar{\xi}_{k} d \bar{S}, \quad i=1,2,3,
$$

in which a summation on $k$ is implied. The quantities $\sigma_{i k}$ form a symmetric matrix called the stress matrix. It was shown by Cauchy that this matrix does indeed yield the stress distribution at a point in accordance with (2.5). The proof requires the use of Newton's laws and involves a passage to the limit in which $d \bar{S}$ is shrunk down on a point. It has been known for a long time that the quantities $\sigma_{i k}$ form a tensor in the sense of the definition of tensors with respect to their invariant properties under coordinate transformations. As the word tensor itself proves, the stress tensor was the prototype of all tensors - thus pointing out a debt which mathematics generally owes to continuum mechanics.

The next step in the development of continuum mechanics consists in deriving the equations of motion. These are simply the expression of Newton's law, together with the assumed existence of a tensor stress field $\sigma_{i k}$ which is differentiable. These equations, the expression of the law of conservation of momentum, are

$$
\bar{\rho} \frac{\partial^{2} \bar{x}_{i}}{\partial t^{2}}=F_{i}+\frac{\partial \sigma_{i k}}{\partial \bar{x}_{k}}, \quad i=1,2,3 .
$$

Here $\bar{\rho}$ represents the mass per unit volume in the deformed position fixed by $\bar{x}_{i}$, and the quantities $F_{i}$ represent the external forces per unit volume (which arise through immersion of the body in a field of force such as gravity). The summation convention on the repeated index $k$ is again to be observed. The law of conservation of angular momentum is not written down: it, in fact, leads simply to the symmetry of the stress tensor, as Cauchy showed. Since Newton's laws are of necessity formulated with respect to the instantaneous position of a particle, it is inevitable that the force resulting from the space gradients of the stress tensor field must be computed in the deformed position and hence this requires that partial derivatives with respect to the variables $\bar{x}_{i}$ must be taken. These last are, on the other hand, clearly unknown functions to be determined in general as part of the solution of a specific problem. This is one of the main reasons why the Lagrange variables employed here are avoided in the great bulk of the literature in continuum mechanics. However, in elasticity, as we shall see shortly, this difficulty is not fatal-in fact, the theory employing the Lagrange variables is rather natural in this field. 
We have now in our possession the main elements of the general mechanics of continuous media. We owe it in the main to Cauchy, although the way had been prepared by the Bernoullis and Euler. Little more can be done without making hypotheses about the medium. In fact, the equations (2.6) are only three in number, but they contain ten unknown functions, in general, if the body force components $F_{i}$ are regarded as given: the density $\bar{\rho}$, the three coordinates $\bar{x}_{i}$ defining the position of the system, and the six components $\sigma_{i k}=\sigma_{k i}$ of the stress tensor. Thus more relations are needed, and these arise by way of mathematical formulations based on the assumed physical properties of the various types of media.

For example, one of the simpler cases from this point of view is furnished by what is called a perfect incompressible fluid. The word "perfect" in this connection refers to the nonexistence of viscosity, and this in turn is interpreted to mean that no shear stresses can occur; as a result the stress matrix $\sigma_{i k}$ is given by $-\sigma 1$, i.e. it is a diagonal matrix with $-\sigma$ as the common scalar value of its elements. The quantity $\sigma$ is called the pressure in the fluid. The stress field thus reduces to a scalar field, and equations (2.6) then contain only four unknown functions, i.e. the functions $\bar{x}_{i}$ and $\sigma$, since $\bar{\rho}=\rho$. The condition of incompressibility is formulated in the obvious way by requiring the determinant of the Jacobian matrix $p_{i k}$ to have the value unity; thus a fourth equation is made available. These equations together with appropriate initial conditions (which fix the position and velocity of all particles at the time $t=0$ ) and boundary conditions would constitute a fully formulated problem in mechanics.

Another possibility is that furnished by elastic solids. In this case the hypothesis could reasonably be made that the stress components $\sigma_{i k}$ at a point are given functions of the elements $\eta_{i k}=\eta_{k i}$ of the strain matrix $\eta$ defined by (2.4), which in its turn is a given function of the unknown first derivatives of the coordinates $\tilde{x}_{i}$ in the deformed position. These relations could be substituted into (2.6) in place of the functions $\sigma_{i k}$, with the result that the three equations would involve only the three unknown quantities $\bar{x}_{i}$. Again, appropriate initial and boundary conditions would arise from special physical conditions which might be imposed-for example, the whole boundary of the solid might be prescribed to be held fixed.

It requires very little experience with problems involving partial differential equations to realize that the two kinds of problems in mechanics which we have just outlined lead to mathematical problems of very great difficulty and complexity. However, solutions of them-and of many others of far greater difficulty in continuum 
mechanics-are very much desired in practice since the physical theories of which they are the mathematical formulations have been known for a century or more to be capable of mirroring the phenomena of nature with good accuracy in many highly important special cases. Thus mathematical techniques for their solution are wanted, since that would make largely unnecessary a great deal of expensive and inefficient experimentation and trial and error methods.

It is perhaps not superfluous to say what is meant by "solutions" of our problems, and what that implies. From a strictly mathematical point of view it could be regarded as sufficient to establish, if possible, the existence of functions $\bar{x}_{i}\left(x_{1}, x_{2}, x_{3}, t\right), \sigma_{i k}\left(x_{1}, x_{2}, x_{3}, t\right)$, etc. which have derivatives of a certain order and which satisfy all of the differential equations and boundary and initial conditions identically. One would also like to know whether or not such a solution is uniquely determined. It seems highly probable that the specialists in mechanics have on the whole formulated their problems in such a sensible fashion, and with such correct instincts, that the solutions do exist in this sense, but rigorous proofs of such plausible conjectures are altogether lacking for problems formulated in the generality indicated above by way of example in the cases of perfect fluids and perfectly elastic bodies. One has a few such proofs only for very special and relatively simple cases. As an indication of how this matter stands, I point out that the Dirichlet problem and Plateau's problem, both of which occur as particularly simple problems in elasticity, have been solved in the sense indicated here, but only after long struggles and great efforts on the part of some of the best mathematicians of our time, who also were forced in the process of doing so to invent entirely new methods of analysis which in their turn resulted in new and flourishing branches of mathematics of a pronouncedly abstract character. And yet, these two problems are rather easy when compared with those in view here.

The problem of proving the uniqueness of the solutions of our mathematical problems is also not easy to solve, even when it happens to be true, which is often simply not the case. In fact, it is my intention in this lecture to deal largely with problems in which the nonuniqueness of certain types of solutions is one of the central points of interest.

For practical purposes one wants more than the assurance that the mathematical problem is not made nonsensical by reason of a formulation which is contradictory because no solution of it exists. What one wants and needs in practice is a representation of the solution, as explicit as possible, which is in such a form that not only its depend- 
ence on the independent variables $x_{i}$ and $t$ is effectively obtained, but also is such that its dependence on all relevant physical parameters over their appropriate ranges can be read off. This is a very large order. All of the resources of mathematical analysis as we know it, intricate, subtle, varied and complicated though they are, are puny and quite insufficient to cope with such problems in their full generality. I hasten to add, however, that the practitioners in the field have, nevertheless, accomplished great things by narrowing the formulations, making a host of tactful simplifying assumptions, restricting the physical parameters to very small values (or, on occasion, to very large values), and then attacking the problems with the whole artillery provided by mathematical analysis. Some idea of what I mean will, I hope, come out in the course of my discussion later on of problems in a small part of the general field of continuum mechanics.

There is an important aspect of these last matters which deserves at least a mention here. I refer to the existence of high speed digital computing machines and the constant development of new and ever faster ones, which, according to a widespread and quite erroneous impression, should make it possible to solve numerically the problems of continuum mechanics and thus make the observations of the preceding paragraph needlessly pessimistic. J. von Neumann, who was the pioneer in the development of this kind of computing equipment, did so with the object of solving numerically certain types of problems in continuum mechanics, and he was extraordinarily successful in that enterprise. We have at our disposal now, to a large degree because of his efforts, computing equipment which allows us to attack successfully problems which only a few years ago would have been regarded as hopelessly difficult. However, the problems solvable in this fashion are, again, relatively simple problems. For example, if the problem depends on more than two independent variables $\left(x_{1}, x_{2}, x_{3}\right)$ say, or $\left(x_{1}, x_{2}, t\right)$ (not to mention the most general case $\left.\left(x_{1}, x_{2}, x_{3}, t\right)\right)$, then it is highly unlikely that its solution could be approximated numerically by the best and fastest available computing equipment-partly because present methods would require formulations involving nets of points in three or four dimensional space. But it would be a mistake to think that the main difficulty lies in the capacity, or rather lack of capacity, of machines to perform calculations with lightning speed, to have a prodigious memory in which to store needed numbers, routines, etc. The difficulties lie rather in the domain of human capabilities. In the use of machines for solving problems of the type under discussion here, i.e. complex nonlinear problems formulated in terms of partial differential equations, it is 
absolutely vital to master first of all the underlying mathematical theory of such problems, and then on that as a basis to derive a numerical scheme that will effectively yield an accurate approximation to their solution. In fact, a new and important branch of analysis called numerical analysis has resulted from these needs.

3. Elasticity. In the preceding section it was pointed out that the general formulations of continuum mechanics are incomplete until relations arising from physical hypotheses about the medium are introduced. In the case of elastic solids it was observed that these could take the form of six explicitly given equations for the stresses $\sigma_{i k}$ as functions of the strains $\eta_{i k}$, and such relations should characterize the medium physically. This is the course followed in the classical linear theory of elasticity, in which these relations are all taken to be linear and homogeneous equations connecting stress and strain, on the basis of the assumption that the strains are all infinitesimals of first order. In this lecture, however, it is not appropriate to make such a special assumption, and it is then preferable to formulate the general theory in a different way.

The theory goes back basically to Kirchoff, according to C. Truesdell, and its main assumption is that the elastic properties of a given material can be described adequately by assuming that a strain energy density function exists which depends only upon the strains induced by a given deformation. Most of the more recent writers in the field make use of this hypothesis. ${ }^{1}$ The general custom is to develop the theory in an invariant manner by making use of the tensor notation, which leads to a rather bewildering maze of formulas and a thicket of subscripts and superscripts. The mathematical formulation followed in this lecture is essentially that of Murnaghan [27], who bases his treatment of the theory of matrices, and also works in principle with the Lagrange variables; he thereby simplifies the formal aspects of the theory quite materially, in my opinion, and at the same time presents them very clearly and concisely.

The basic assumption of this form of the theory of elastic solids is that there exists a strain energy density function $\mathcal{u}(\eta)$, defined with respect to the undeformed and unstrained stage of the elastic body, which, as indicated above, depends only upon the strain matrix $\eta$ of the deformation. Of course, the elements of $\eta$ are, in their turn, func-

1 The theory is derived and treated at length in the book by Green and Zerna [13]. For a history and bibliography of the literature, which is very extensive, the papers [42] and [43] by C. Truesdell should be consulted. Particularly relevant for the present lecture are papers by Biot [2;3], Kappus [20], Rivlin [35], Reissner [33], Trefftz [40], the classic book of Love [25], and the book of Novozhilov [28]. 
tions of the elements of the Jacobian matrix $p$ of the deformation. All relevant physical properties of the elastic solid are supposed to have their appropriate formulation in this function. Thus we have

$$
u=\mathfrak{u}(\eta) \equiv \mathfrak{u}\left(\eta_{i k}\right) .
$$

It is assumed next that the rate $d W / d t$ at which work is done on the body by the body forces $F_{i}$ and the stresses applied at its boundaries is equal to the rate of change $d K / d t$ of the kinetic energy plus the rate of change $(d / d t) \iiint_{K} \mathcal{U} d V$ of the strain energy of the body:

$$
\frac{d W}{d t}=\frac{d K}{d t}+\frac{d}{d t} \iiint_{R} u d V .
$$

Here $R$ represents the domain occupied by the unstrained body. The variational principle of virtual work is then applied in order to obtain both the equations of motion and the various possible types of boundary conditions which are permissible. In doing so, it is of advantage to introduce a new set of functions $q_{i r}(\eta)$ which are defined by the equations

$$
q_{i r}(\eta)=\frac{\partial \mathcal{U}(\eta)}{\partial p_{i r}} .
$$

These functions are, as we shall see in a moment, quantities which are of the nature of stresses, but unlike the stresses $\sigma_{i k}$, which are defined in the deformed position of the body, they are evidently defined in the undeformed position, i.e. with respect to the Lagrange coordinates. Also, the matrix $q \equiv\left(q_{i r}\right)$ is not usually symmetric. The variational methods now lead in standard fashion to the following equations of motion:

$$
\frac{1}{|p|} \frac{\partial q_{i r}}{\partial x_{r}}+F_{i}=\bar{\rho} \frac{\partial^{2} \mathbf{x}}{\partial t^{2}}, \quad i=1,2,3,
$$

and these equations have the advantage, as compared with (2.6), that no derivatives with respect to the dependent variables $\bar{x}_{i}$ occur. By the symbol $|p|$ we mean the Jacobian determinant $\left|p_{i k}\right|$. We recall that a summation on the index $r$ is implied in (3.4). We see also that the terms in (2.6) involving the stresses $\sigma_{i k}$ are replaced in (3.4) by terms involving the "Lagrange" stresses $q_{i k}$. In applying the variational principle it is, as usual, necessary to restrict the variations to those which are compatible with any geometrical constraints that might be prescribed at the boundary of the solid. If no such constraints are imposed, then so-called natural boundary conditions 
arise, and these take the form of conditions involving the stresses at the boundaries. One form of these conditions turns out to be the following: ${ }^{2}$

$$
\sigma_{i k}=\frac{1}{|p|} q_{i r} p_{k r}=\frac{1}{|p|} q p^{*} .
$$

As one sees, these are relations between the stresses $\sigma_{i k}$ and the functions $p_{i k}$ which determine the strains. They are, in fact, a replacement for the stress-strain relations of the classical theory. The fact that they were derived as boundary conditions is not important in this connection: any subdivision of the original body could be considered just as well as the whole in applying the variational principle. An equivalent formulation of (3.5) is the following:

$$
q_{i r} \xi_{r} d S=\sigma_{i k} \bar{\xi}_{k} d \bar{S},
$$

in which $\xi_{k}$ and $d S, \bar{\xi}$ and $d \bar{S}$ represent the unit normals and the elements of area in the original and the deformed positions. The conditions (3.6) are useful in practice in formulating the boundary conditions-for example, an unstressed portion of the boundary is characterized by $\sigma_{i k} \bar{\xi}_{k}=0$, and hence $q_{i r} \xi_{r}=0$ on its pre-image; thus a boundary condition is obtained for a free boundary, but with respect to the undeformed position, and this is a great advantage in such cases.

This is so far an extremely general theory. We now specialize quite drastically by assuming the solid to be isotropic, that is, that its properties are not dependent on direction at any point, and that it is also homogeneous, i.e. its elastic properties are the same at all points. It follows that the function $\mathfrak{u}(\eta)$ must depend only on invariants of the strain matrix $\eta$ (which might be formulated either by (2.4) or $(2.4)^{\prime}$ ) with respect to orthogonal coordinate transformations, and these are, of course, the coefficients of the cubic equation which determines the eigenvalues of the strain matrix $\eta$. In his work Murnaghan assumed the strain energy density to be developable in powers of these invariants, and he investigated in a number of cases (for example, in cases of constant stress, or of strains given as linear functions of the coordinates) what conclusions might be drawn by taking a few terms in such a series, with coefficients to be determined from experiments, upon extrapolation to pressures so high as to be beyond the range of experimentation.

${ }^{2}$ This relation can be rather more directly obtained by equating the work done by the stresses on a volume element to the change in strain energy which arises from an arbitrary variation in the displacements. 
Here, for the sake of concreteness, we might introduce for the strain energy density the expression:

$$
\mathcal{u}=\frac{\lambda}{2}[\eta]^{2}+\mu\left[\eta^{2}\right]
$$

with $\eta$ the matrix given by $(2.4)$ or $(2.4)^{\prime}$, and with the symbol $[A]$ meaning the trace of the matrix $A$. Since the body is supposed to be homogeneous it follows that $\lambda$ and $\mu$ are constants for a given material, presumably with values which would differ depending upon which of the two definitions is taken for the strain matrix $\eta$. The constants $\lambda$ and $\mu$ might be called the Lamé constants, since (3.7) reduces to one of the standard formulas for the strain energy in the classical linear theory of elasticity. Figuring in (3.7) are, evidently, the linear invariant $[\eta]$ and the quadratic invariant $\left[\eta^{2}\right]$ of the matrix $\eta$; the cubic invariant $|\eta|$, which could occur, is not employed here. The function defined by (3.7) reduces, as I have said, to the form used in the classical linear theory of elasticity when it is assumed that the strains and deformations are very small. In what follows, however, it is not at all essential to suppose that $\mathfrak{U}$ has the special form given in (3.7).

With the introduction of a specific strain energy density function, the theory of elasticity can now be said to be complete.

So far, I have supposed that the states considered were time dependent, since that causes little or no complication in outlining the basic theory. From now on, however, I concentrate on equilibrium states with no body forces present. In that case, the equations of motion (3.4) simplify greatly; they become, in fact

$$
\frac{\partial q_{i k}}{\partial x_{k}}=0, \quad i=1,2,3 .
$$

This is a set of three equations for the nine functions $q_{i k}$, since the matrix $q_{i k}$ is, in general, not a symmetric matrix. To this, however, one may add the nine defining equations (3.3) for these quantities. We would therefore have twelve equations for the nine functions $q_{i r}$ plus the three functions $\bar{x}_{i}$, and thus twelve equations for the twelve unknown functions; or, one could, in principle, replace the functions $g_{i r}$ in the set of equations (3.8) in terms of the functions $\eta_{i r}$ which, in turn, are given in terms of first derivatives of the displacements $\bar{x}_{i}$. In that way, a system of three nonlinear partial differential equations of second order for the functions $\bar{x}_{i}$ would be obtained. To these equations must be added appropriate boundary conditions which might be expressed in terms of stress, or of displacement, or both. The analytical problem of determining functions $q_{i k}$ and $\bar{x}_{i}$ which satisfy 
the differential equations and appropriate boundary conditions is, as has already been said, an extremely difficult, complex problem in spite of the extreme simplicity of the equations (3.8) in outward appearance. Such problems are very difficult even if one is prepared to be contented with a numerical approximation.

What then is one to do? The answer is rather obvious: it becomes necessary to take advantage of possibilities which may result through simplifying and specializing the physical problems; for example, the vast bulk of the literature in the field of elasticity is in the domain of the classical linear theory of elasticity, which results when one assumes that the strains as well as the deformations are small. The equilibrium problems then become linear problems for elliptic partial differential equations. Of this theory I do not want to speak here. In fact, I have already said that I intend to deal mainly with problems concerning the stability of an elastic solid in equilibrium, and these are problems which are, in principle, not linear.

4. Stability of thin solids under pressure. The prototype of all problems in elastic stability is the problem of buckling of a long slender elastic column or rod under compressive forces at its ends and along its axis. This problem was treated some two hundred years ago by Euler. The physical occurrence is quite easy to explain and to understand. Obviously, if the column is long and slender, the straight unbent state of it will not remain stable if the end compression is made too large: that is clear to everyone's physical sense. What happens is for our purposes perhaps most clearly explained schematically by considering how the maximum deflection $w_{\max }$ depends on the end thrust $F$ (cf. Figure 4.1). For $F$ sufficiently small,

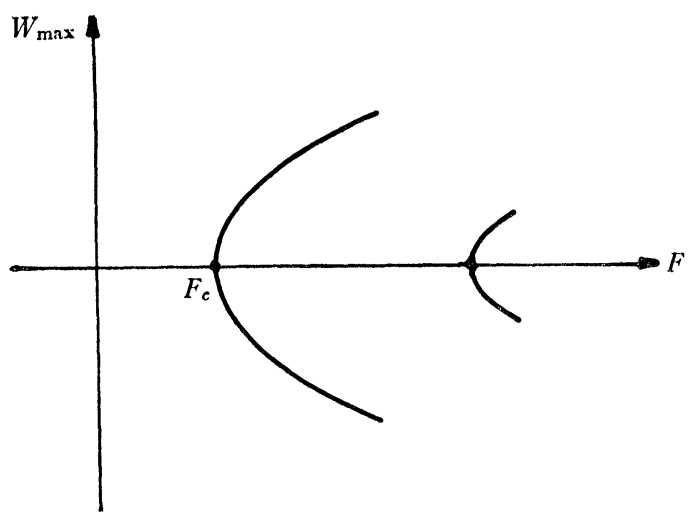

FIGURE 4.1. Bifurcation phenomena. 
nothing happens, $w_{\max }$ is zero, and the straight state is the only equilibrium state. At a certain critical value $F_{c}$ of $F$, however, the straight state ceases to be the only possible equilibrium state and a bifurcation of the solution of the equilibrium problem takes place; the bent state is then the stable state of equilibrium. This is truly a bifurcation phenomemon in the sense meant by Poincaré; that is, $w$ is a function $w(x, F)$ of both the independent variable $x$ and of the load parameter $F$ such that when $F$ attains the critical value new types of solutions of the equilibrium problem appear in the neighborhood of the unbent state which co-exist for the same values of the physical parameters. Quite generally, when I speak here about instabilities, it is this type of phenomenon that I have in mind, namely the coexistence of more than one equilibrium state in an arbitrary neighborhood of some given state. Of course, many of you are familiar with what is really true in the present case, i.e. that there are infinitely many critical values and correspondingly many different buckled shapes or modes of the column. In fact, this problem of the determination of the critical loads and buckling modes is, I believe, historically the first example of a linear eigenvalue problem associated with a boundary value problem for differential equations. The differential equation is $d^{2} w / d x^{2}+\lambda w=0$, with $w(0)=w(l)=0$ as boundary conditions and with $\lambda$ proportional to the square root of the axial force $F$ : it is, as one sees, indeed the prototype of all eigenvalue problems associated with differential equations.

It can now be made obvious why it is that the theory of elastic stability cannot belong to the classical linear theory of elasticity, since the boundary value problems of that theory, when properly formulated, have uniquely determined solutions and here, obviously, that is just not the case. It is true, as I have already said, that the critical buckling values of the axial load $F$ are determined as the eigenvalues of a linear problem; however, this comes about through a perturbation procedure as applied to a basically nonlinear formulation of the relevant problem in elasticity.

The Euler theory for the buckling of a thin rod is not a theory which can be obtained directly from the basic nonlinear theory of elasticity which was formulated earlier. Instead, it results by making a lengthy series of assumptions regarding the elastic behavior of a body with a very special property, namely that one of its dimensions is large compared with the other two. Until recently, the entire theory of elastic stability was confined to cases of this sort, namely to thinwalled solids, such as thin columns, thin plates, and thin shells, and mathematical theories have been derived for these cases which are 
based not on a specialization of an underlying nonlinear three-dimensional theory, but rather on hypotheses regarding what seems likely to happen through the thin wall of the solid.

I proceed to formulate one of the best known and most used of these theories, namely the thin plate theory which is due to Föppl and von Kármán [21]. This theory leads to the following pair of nonlinear partial differential equations:

$$
\begin{aligned}
(\gamma h)^{2} \Delta \Delta w & =-\left(\phi_{y y} w_{x x}-2 \phi_{x y} w_{x y}+\phi_{x x} w_{y y}\right), \\
\Delta \Delta \phi & =\left(w_{x x} w_{y y}-w_{x y}^{2}\right) .
\end{aligned}
$$

We have here two partial differential equations of fourth order, each involving the biharmonic operator (the repeated Laplacian, that is), and with coupling through quadratic terms in second derivatives of two dependent functions $w(x, y)$ and $\phi(x, y)$ which, with the addition of boundary conditions, serve to formulate the problem of buckling of plates under compression along the boundary. The function $w(x, y)$ represents the vertical deflection of the plate (cf. Figure 4.2), while $\phi(x, y)$ is called the Airy stress function, from which the so-called membrane stresses (they are, in fact, average stresses over the thickness) are derived by differentiation in accordance with the following equations:

$$
\sigma_{x x}=\frac{\partial^{2} \phi}{\partial y^{2}}, \quad \sigma_{x y}=-\frac{\partial^{2} \phi}{\partial x \partial y}, \quad \sigma_{y y}=\frac{\partial^{2} \phi}{\partial x^{2}} .
$$

(Actually, these are dimensionless stresses obtained by dividing the actual stresses by Young's modulus.) Thus the originally threedimensional problem is made to depend on only two of the coordinates, or, as one might also put it, the behavior of the plate is referred to its middle surface.

It has no very great point to discuss here in any detail the fairly complicated physical and geometrical hypotheses which lead to the differential equations (4.1) and (4.2). One of the hypotheses, however, deserves mention because of its relation to a matter of importance to be discussed in the next section. It refers to the strain components $\eta_{11}$ or $\eta_{22}$ or, in the $x, y, z$ coordinate system of Figure 4.2 , the components $\eta_{x x}$ or $\eta_{y y}$. In the classical theory in which the strains are defined by $(2.4)^{\prime}$ one has for $\eta_{x x}$, for example, the following formula:

$$
\eta_{x x}=u_{x}+\frac{1}{2}\left(u_{x}^{2}+v_{x}^{2}+w_{x}^{2}\right),
$$

when $u, v, w$ represent the displacements, i.e. the differences $\bar{x}-x$, 

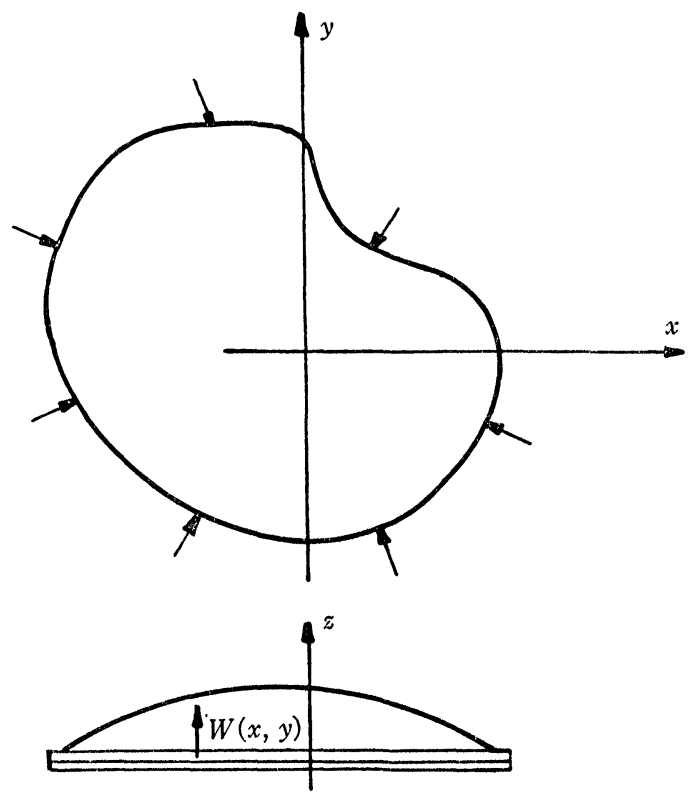

FIGURE 4.2. Buckling of a thin plate.

$\bar{y}-y, \bar{z}-z$ of the coordinates of the points of the solid in the original and deformed positions. One of the key assumptions of the Föpplvon Kármán theory is that the plate, because of its thinness, might be expected to admit of a vertical displacement which is of a higher order of magnitude than the horizontal displacements, without at the same time violating another of the important assumptions, i.e. that the strains should remain small. Thus (4.4) is simplified very greatly by dropping the terms $u_{x}^{2}$ and $v_{x}^{2}$, but retaining the term $w_{x}^{2}$; i.e. the strain component $\eta_{x x}$ is assumed to be given approximately by

$$
\eta_{x x}=u_{x}+\frac{1}{2} w_{x}^{2}
$$

This nonlinear relation is the source of the nonlinearities in the equation (4.2), and it is in a way the key assumption of the theory. More will be said later about the relation between (4.4) and (4.5).

While it is true that these differential equations represent a very great simplification by comparison with the general three-dimensional nonlinear theory-after all, there are only two independent variables and two dependent variables left-nevertheless, it should be quite obvious that the boundary value problems which remain to 
be solved are very formidable problems indeed. (One gets an idea, then, of the complexity to be expected in the general nonlinear theory if it were to be formulated in extenso in differential equations.) They are such that nothing very much of a general nature can be done; for example, solutions given explicitly by series or by integral representations are more or less out of the question. Once more, simplifications are in order, if it is desired to discuss any concrete problems analytically.

One of the favorite ways of formulating attackable problems in mathematical physics will now be employed, namely the device of assuming the problem to have certain symmetries. In this case, it is fruitful to suppose that one is dealing with a circular plate which is bent into a form with symmetry with respect to the center, and hence such that the dependent functions can be supposed to depend only upon the radius $r$ (see Figure 4.3). It is convenient to introduce two new dependent functions $q(r)$ and $\sigma(r)$ defined in terms of $w$ and $\phi$ by the equations

$$
\begin{aligned}
& q(r)=-\frac{R}{r} w_{r}, \\
& \sigma(r)=-\frac{1}{r} \phi_{r},
\end{aligned}
$$

in which $w_{r}$ and $\phi_{r}$ denote derivatives with respect to $r$. Evidently $q(r)$ measures the radial slope of the plate. The function $\sigma(r)$ is the radial stress, the so-called radial "membrane" stress, indicated in Figure 4.3. In terms of these quantities the differential equations
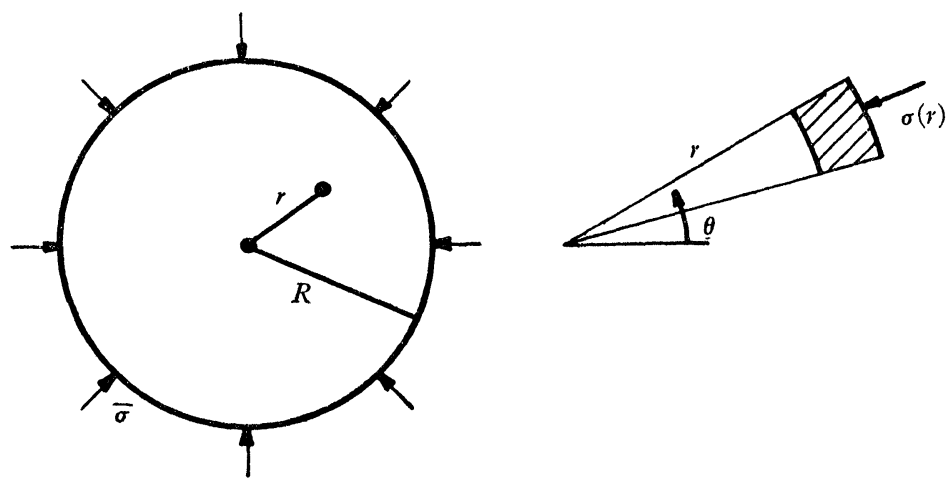

FIGURE 4.3. The circular plate. 
(4.1) and (4.2) can be very much simplified and reduced in order; the differential equations become, in fact:

$$
\begin{gathered}
G \sigma-\frac{1}{2} q^{2}=0, \\
\kappa^{2} G q+\sigma q=0,
\end{gathered}
$$

in terms of the linear differential operator $G$ defined by

$$
G()=R^{2} r^{-3} \frac{d}{d r}\left(r^{3} \frac{d}{d r}()\right),
$$

with $\kappa=r h / R$ a parameter involving the material constant $\gamma$ and the ratio $h / R$ of thickness to radius of the plate. To these differential equations we add the following boundary conditions:

$$
\begin{gathered}
\sigma_{r}=q_{r}=0 \text { at } r=0, \\
\sigma=\bar{\sigma}>0, \quad R q_{r}+(1+\nu) q=0 \text { at } \quad r=R .
\end{gathered}
$$

The conditions (4.11) are conditions of regularity at the center $r=0$ of the plate which are appropriate since $G$ is singular there. The first condition in (4.12) states that the edge compressive stress is $\bar{\sigma}$ (and counted positive when it is a compression), while the second condition corresponds to the assumption that no constraint is imposed at the edge to inhibit turning of the plate about the tangent; or, expressed in a different way, one says that the plate is simply supported there. The mathematical problem to be solved is then to determine functions $\sigma(r, \bar{\sigma}), q(r, \bar{\sigma})$ which satisfy the equations (4.18) to (4.12) for all values of the applied compressive force $\bar{\sigma}$ at the boundary, i.e. for $0 \leqq \bar{\sigma}<\infty$.

This is a problem which was solved some twenty years ago by K. O. Friedrichs and the speaker [10]. It is possible to explain here how that was done only in very general terms. For values of $\bar{\sigma}$ not too large it was found that the solution could be obtained by means of power series in the independent variable $r$, and this was supplemented by an asymptotic development with respect to large values of $\bar{\sigma}$. Fortunately, it happened that the two forms of the solution overlapped. I might remark that Friedrichs and I spent practically all of our spare time on numerical computation for a year or more in order to obtain actual numerical solutions over the whole parameter range, since only desk computers were available at that time. It is perhaps a measure of the progress that has been made in this area to observe that this same problem was attacked recently in our Institute with a modern digital computer; it proved to be more or less a triviality for 
such a machine since very accurate results were obtained in a matter of minutes.

I want next to describe briefly the results which were found for the problem formulated above, since they are characteristic for this type of problem, and they have general connotations. To begin with, the whole matter is of much the same sort as was described for the case of Euler's column problem. The boundary value problem just now formulated has as one of its solutions the solution $\sigma \equiv \bar{\sigma} ; q \equiv 0$, which means that the plate remains plane and in a state of uniform compression-no buckling occurs. This clearly satisfies the differential equations as well as the boundary conditions. Critical values for the boundary pressure $\bar{\sigma}$ at which other solutions appear can be obtained here also by solving a linear eigenvalue problem. For that purpose one supposes $q$ to be small, say of first order, while $\sigma$ is supposed to be given by $\bar{\sigma}$ plus a small term of first order. These assumed solutions are inserted in the differential equations, and it is evidently natural to ignore $q^{2}$ in the first equation and to replace $\sigma$ in the second equation by $\bar{\sigma}$. This latter equation then is a linear homogeneous equation for $q$, which in conjunction with the linear homogeneous boundary condition given in (4.12) poses a linear eigenvalue problem with $\bar{\sigma}$ as parameter. One then asks the question whether values of $\bar{\sigma}$ exist such that nonidentically vanishing solutions $q(r)$ arise. There exists a whole spectrum of such values; in fact, we have here a particular case of the classical Sturm-Liouville eigenvalue problem with an infinite discrete spectrum and corresponding eigenfunctions (they are Bessel functions), each one of which yields a possible mode of buckling.

Since the linear buckling problem is homogeneous, it follows that the buckling modes are not determined in amplitude, but only within an arbitrary constant multiplying factor. In order to determine deflections and stresses after buckling occurs, it is then necessary to integrate the nonlinear differential equations. As I have said, we did that in the sense that we followed the first branch of the bifurcation curve (that is, the branch that goes out from the lowest critical buckling value, ${ }^{3}$ see Figure 4.1 with $F$ and $F_{c}$ replaced by $\bar{\sigma}$ and $\bar{\sigma}_{c}$ ), and indeed for the whole possible range $0 \leqq \bar{\sigma}<\infty$ of the applied compressive load.

It is of interest to describe briefly the results we obtained for some

${ }^{8}$ In his doctoral thesis W. Koiter [22] has studied in detail the nature of the bifurcation curves in the neighborhood of a bifurcation point in a variety of cases concerning thin curved shells; in these cases the phenomena are a good deal more complicated than they are in the present case. 
of the quantities. For example, I show a curve (see Figure 4.4) for the radial compression $\sigma$ at the center $r=0$ of the plate as a function of the pressure $\bar{\sigma}$ applied at the edge, all other parameters being held fixed. As one sees, an increase in the normal pressure at the edge results after buckling in a decrease in the value of the radial stress at the center of the plate. The reason for that can be understood by an

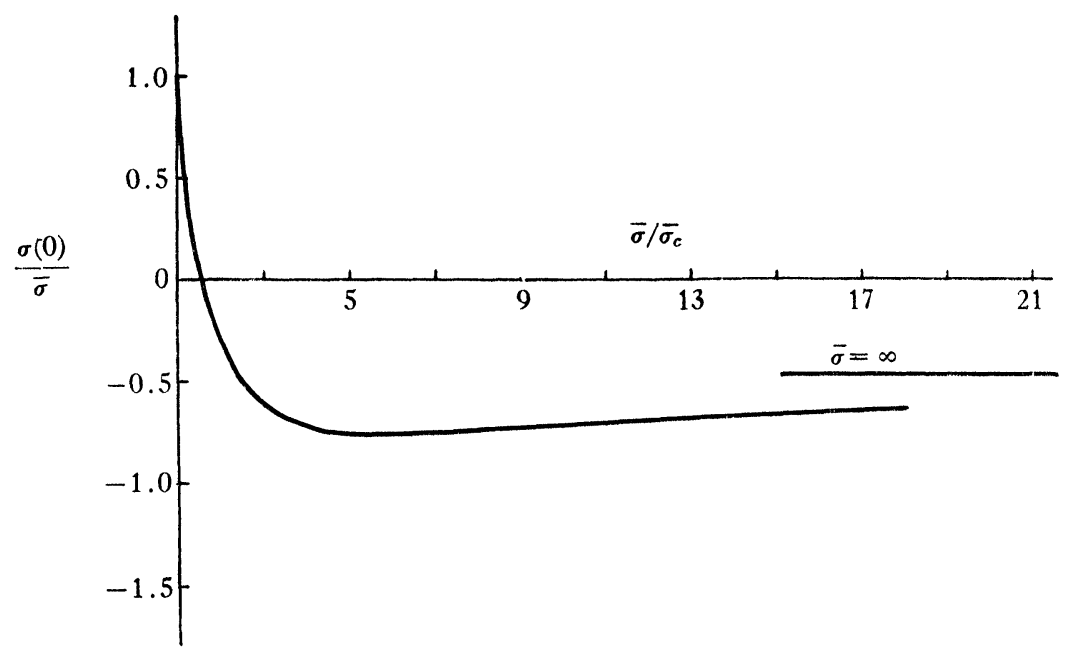

FrguRE 4.4. Radial membrane stress at center.

appeal to differential geometry. If a plane surface is held fixed at its edges but is then deflected out of the plane it will in general be stretched. In the present case, the stretching of the plate due to its bulging out after buckling results in tensile strains in the plate and, consequently, to a partial relief of the original compressive stress in the flat plate. As the curve indicates, this process goes so far that it eventually, under sufficiently high pressure at the edge, leads to a change in the sign of the radial stress, which means that it goes over into a tension. When we first obtained this result we thought we must have made a mistake, since at first sight it seemed hard to believe. However, the solution of the mathematical problem has this property (and such a behavior of buckled plates is also well known experimentally). I might add that in any event rigorous proofs of this and of other statements of mathematical character that are made below are contained in the paper cited above.

It will be observed that the curve for the radial stress at the center has a horizontal asymptote. This asymptotic value was determined by calculation from an appropriate asymptotic theory. Before saying 


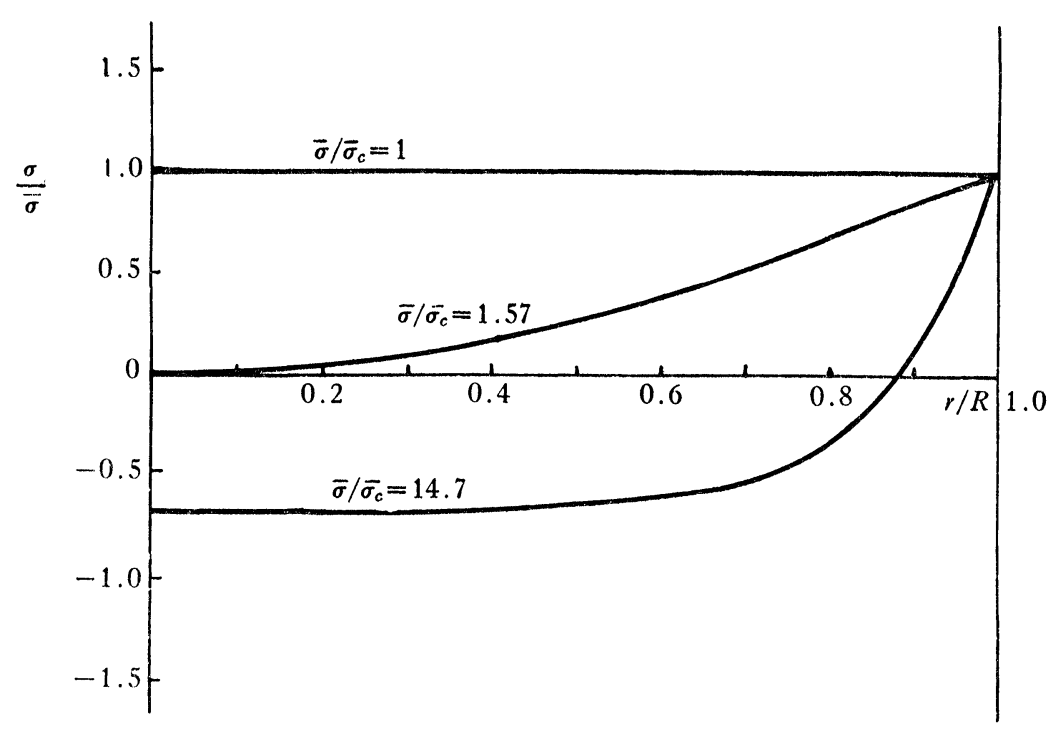

FIGURE 4.5. Radial membrane stress.

more about that, however, I show another set of curves (see Figure 4.5). These curves represent the behavior of the radial stress, but this time as a function of the radius, and various curves are given for different values $\bar{\sigma} / \bar{\sigma}_{c}$ of the ratio of the applied pressure at the edge to the lowest critical pressure. Since, as we have already seen, the stress at the center changes its sign as the compression at the edge increases - that is, it goes over into a tension-it is not surprising that the curves for $\sigma$ as a function of $r$ have the general form shown on the figure, in the sense that the part of the plate over which this stress is a tension gradually spreads outward from the center towards the edge as $\bar{\sigma}$ increases. The curves indicate, however, much more than that, namely that this stress seems to be tending to a constant negative value in the inner part of the plate and then to change rapidly near the edge of the plate to the prescribed positive value at the boundary. In fact, the limit situation is one in which the entire interior of the plate is in a state of constant tension, with a discontinuous change from that negative value in the interior to the prescribed positive value at the boundary. All physical quantities change in this way, in fact, i.e. all of them tend to be constant in the interior of the plate and to change very rapidly in a narrow strip or boundary layer near the edge of the plate. It was this conception of the behavior of the solution of the nonlinear boundary value problem, obtained as the result of numerical computation, which led to the proper formu- 
lation and to the solution of the asymptotic problem appropriate for $\bar{\sigma}$ very large.

It is indeed a typical boundary layer phenomenon which is involved here, and the mathematical reason for its occurrence is seen by examining the differential equations. Consider first the differential equation (4.9) for $q$. In it we expect $\sigma$ to become large since it is so prescribed at the boundary; thus, one might expect in the limit that the first term of (4.9) could be ignored compared to it. Perhaps a better way to put it would be to say that the limit state characterized by letting $\bar{\sigma}$ become infinite while other parameters are held fixed could be characterized just as well by holding $\bar{\sigma}$ fixed but allowing the thickness of the plate to approach zero. In the latter case, as one sees, the parameter $\kappa$ would tend to zero and thus in this way also one sees that the limit equation resulting from (4.9) should be $p q=0$, from which we conclude that $q \equiv 0$. But if $q \equiv 0$, it is readily seen that the only solution of the equation (4.8) for $\sigma$ which is regular at the center is $\sigma \equiv$ const. Thus we would have indeed in the interior of the plate the limit state $q \equiv 0$, that is, the plate would become flat, and also $\sigma$ would be a constant. Both of these expectations were borne out by the numerical computations. However, it would evidently be wrong to take as the constant value of $\sigma$ its value $\bar{\sigma}$ at the boundary, since we know that the limit value for this quantity in the interior of the plate is negative, not positive. To find the proper limit value of $\sigma$ in the interior as well as the limit values of all other quantities it is necessary to consider what happens in the boundary layer in the vicinity of the edge of the plate.

Such boundary layer phenomena are not confined to the sort of problem considered here. In fact, the first such problem to be formulated and solved was the famous boundary layer problem of Prandtl [31] which arises in dealing with the flow of a fluid with small viscosity $\nu$ along a rigid boundary: here also the limit solution for $\nu \rightarrow 0$ in the interior of the fluid is obtained by working with the differential equations for $\nu=0$, but it is necessary to analyze what happens in a narrow strip adjacent to the boundary in order to obtain limit values for all of the essential quantities. E. Reissner [34] has recently uncovered an interesting case in the theory of thin elastic shells which gives rise to a boundary layer within a boundary layer. Boundary layer phenomena for thin plates in cases other than that of the circular plate have been treated by Fife [7].

All such boundary layer problems have a common feature: the differential equations are decreased in order when a certain parameter takes on its limit value (in the buckling problem described here the 
order of the system is reduced from four to two when $\kappa=0$ ). As a consequence, the solution of the limit problem cannot in general be expected to satisfy all of the boundary conditions prescribed for the original problem; thus, as in the case described here, the solution of the boundary value problem does not converge uniformly at the boundary when the parameter tends to its limit value. One way to resolve this difficulty is to introduce a new independent variable and also new dependent variables which depend on the parameter in such a fashion that the differential equations in the new variables do not degenerate in order in the limit, and lead to a representation which does converge uniformly at the boundary. This device for obtaining asymptotic representations in degenerate cases is one of many types of asymptotic procedures discussed by Friedrichs [9] in his Gibbs lecture some years ago.

In closing this section it is perhaps of interest to mention two stability problems concerned with the thin circular plate which differ from the one just treated. The first deals with the simply supported circular plate which is bent by uniform normal pressure on its upper face so that its mean surface becomes a surface of revolution. Since the circumference of the plate shrinks in this process, it follows that circumferential compressive stresses (called sometimes hoop stresses) develop near the boundary and it is reasonable to ask whether such stresses might not for high enough loads lead to the instability of the symmetric bent form if nonsymmetric variations are permitted. This turns out to be true, as has been shown by Yanowitch [45], who makes use of the asymptotic solution of the bending problem obtained by Bromberg [4]. A similar question, but for the symmetric buckled states of the plate considered above, has also been answered in the affirmative by Yanowitch-again this comes about because of the development of large compressive hoop stresses near the boundary. Thus the symmetric buckled states of the circular plate may buckle again if the pressure is large enough and the constraint implied in the assumption of symmetry is relaxed.

5. The stability of thick solids under pressure. I have already said that the theory of thin plates as embodied in the differential equations of Föppl and von Kármán is not a theory obtained by direct specialization of a general nonlinear three-dimensional theory. Instead, it results by making a considerable number of special assumptions, the validity of which has never been proved. There is no doubt that this approximate theory has validity in some appropriate mathematical sense. It is, I believe, the lowest order approximation in a development with respect to the thickness of the plate as a small parameter. 
However, such a development is certainly not a convergent but rather an asymptotic development, simply because of the fact that the lowest order approximation involves differential equations which have an order smaller than that of the original differential equations, so that boundary conditions are lost and hence the solution of the basic three-dimensional problem in its dependence on the thickness as parameter will not in general converge uniformly at the boundaries when the thickness tends to zero. In other words, the situation is like that of the limit case of the circular plate discussed above, only that now it is the Föppl-von Kármán equations themselves which would be the degenerate limit differential equations.

Formal developments with respect to the thickness of thin plates have been obtained, for example, by Chien [6], Synge and Chien [39] and, more recently, by Fritz John [19]. Synge and Chien proceed by making formal developments in powers of the thickness, John by assuming double power series with respect to both the thickness and the coordinate in the direction of the thickness. Both procedures lead to a variety of different sorts of approximate theories, depending upon the number of zero coefficients that are assumed at the beginning of the series for the various dependent quantities; among them is the theory of Föppl and von Kármán, the elastica theory of Euler, the so-called membrane theory of shells, and others. There is a challenging problem here: to prove that these approximate theories really are correct in the sense that the terms neglected are all of an order in the thickness higher than those retained. This problem would appear to me, however, to be one of very great difficulty.

Although no rigorous justification of such theories as Euler's elastica theory or the Föppl-von Kármán theory has been given, some recent work by John [18] is relevant and interesting in relation to one of the main assumptions made in these theories. One of the basic assumptions, perhaps the most important assumption, in deriving that theory (as we mentioned earlier), is based on the notion that if the body is thin enough it should be possible to deform it and to bend it in space, but without necessarily causing large strains: after all, a mathematical surface has this property. In fact, linear relations between stress and strain are assumed in this theory as a consequence of the assumption of small strain. This raises interesting and purely kinematic questions which belong to differential geometry in the large.

I formulate the problem in the simplest case, namely that of a rectangular solid. Consider first a solid which has all three dimensions of the same order of magnitude $s$. Suppose that it undergoes a topo- 
logical transformation which has continuous first derivatives. We make now the assumption that the changes in length per unit length of all linear elements, or, in other words, the local strains of linear elements, are small quantities of first order uniformly for all points and for all directions, and let $\epsilon$ be an upper bound for these strains. The question then is: is it possible, after an appropriate rigid body motion has been subtracted, that the deformations can be large, though the strains are small? In the paper cited above John has shown that that is not possible. In fact, in this case, a rigid body motion can always be found such that the remaining deformation is always of the order $s \epsilon$, with $s$ a representative length for the side of the solid. However, if one of the three dimensions of the rectangular solid is small, say of length $h$, so that $h / s$, with $s$ again a significant length for the other two sides of the solid, is small, the situation is quite different. In this case it turns out, for example, that if $h / s$ is of order $\epsilon^{1 / 2}$, then a rigid body motion can always be found so that the displacement components in the directions of the sides of finite length are of order $\epsilon$, but the displacement component in the direction of the small side, that is in the direction normal to the faces of the thin plate, is of order $\epsilon^{1 / 2}$. In other words, the displacement component normal to the plate is of a different and higher order of magnitude than the displacement components parallel to the faces of the plate.

This purely geometric theorem clarifies to some extent one of the basic assumptions of the Föppl-von Kármán theory. This assumption was already referred to above in connection with equation (4.5) as an approximate replacement for equation (4.4). In these equations $u$ and $v$ were displacement components in the plane of the faces of the plate and $w$ was the displacement component normal to them. Since the strain $\eta_{x x}$ is assumed to be of order $\epsilon$, it is clear that if $u$ and $v$ are of order $\epsilon$ while $w$ is of order $\epsilon^{1 / 2}$, then equation (4.5) is indeed an appropriate replacement for equation (4.4) since then all terms in it would be of order $\epsilon$, and the neglected terms would be of order $\epsilon^{2}$. At the same time a further insight is gained, i.e. that the displacement $w$, which is really the deflection of the plate, may not be assumed to be large, but rather it must be of the order of the thickness $h$ of the plate, which in turn is of the order $\epsilon^{1 / 2}$. It might be added that experimental evidence obtained by actually bending plates shows that the Föpplvon Kármán theory is in fact not accurate if the deflection exceeds, say, half of the thickness of the plate.

I was careful to say that the geometrical theorems of John serve to clarify, or perhaps it would be better to say, add plausibility to, the basic assumption of the Föppl-von Kármán theory, but not that 
they give a rigorous justification of it, since it is the first derivatives of the displacements which figure in (4.4) and (4.5), and John's results do not refer to the derivatives. In fact, the behavior of the derivatives of the displacements in such "almost isometric" mappings is a much more complicated affair than is the case for the displacements, as is shown by the fact that quite simple deformations can be given explicitly which yield small strains at all points and in all directions, but which admit isolated points where the local rotation is infinite. However, John [19] has also investigated the behavior of the first derivatives of the deformation. The investigation is delicate, and no more than a rough indication of its outcome can be given here. It is that if the strains are uniformly bounded, then the measure of the set of points where the (generalized) first derivatives of the deformation are above a given bound will become exponentially small when the bound for the strains is made small. This result was obtained for thick solids, not for solids which are thin. It has to be modified for thin solids and would then furnish one of the elements involved in a rigorous proof of the validity of the basic assumption of the thin plate theory as embodied in (4.5).

This discussion about thick rectangular solids vs. thin rectangular solids brings up a question which I had had in my mind for many years. No one has any doubt that a long slender rod will eventually buckle under compression. One might ask, however, whether that would be true for a cube which is compressed on two opposite faces but is left unstressed and free to deform on the other four faces. This question was answered a few years ago by S. Lubkin [26].4 The answer turns out to be in the affirmative; the cube does become unstable under sufficiently high pressure, but the strain which results under such a pressure is enormous, of the order $1 / 2$ in fact, which means that the distance between the compressed faces would be reduced roughly to about half the original value. There are some solids, like rubber, for which such deformations are possible without rupture, and hence this result can be of more than purely theoretical interest. Lubkin also treated in the same paper two other much more difficult cases of the same sort. They were the cases of the hollow thick cylinder, and the hollow thick sphere, both under external pressure. These solids, it turns out, also become unstable under sufficiently high pres-

\footnotetext{
4 E. Trefftz [41] developed a nonlinear theory similar to the one in question here, with similar objects in view. This theory was then applied by Kreutzer [24] to study numerically the buckling of slender columns of circular and rectangular cross-sections, with results which show the Euler theory to give correct values for the critical load. Lubkin assumes boundary conditions of a type which make explicit solutions possible for all thickness-length ratios.
} 
sure. But again, if their thickness is not small, the strains resulting from the high pressures required to cause instability are once more very large. These problems were treated not by introducing a series of ad hoc hypotheses (as is done in deriving the thin plate theory) into the underlying general theory; rather, they are treated by taking the full three-dimensional nonlinear theory, and proceeding by making a small perturbation with respect to the very simple symmetrical equilibrium states the stability of which is to be tested. For example, in the case of the cube a uniform pressure over two opposite faces results in a state of homogeneous strain and constant stress throughout the cube-in fact, one finds readily that this simple state satisfies all of the conditions of the exact nonlinear theory. A small perturbation is then made, and one neglects all but the linear terms in the perturbations. ${ }^{5}$ Or, as one could also put it, the variational equations relative to the basic equilibrium state are formed. The result is a linear homogeneous boundary value problem with the perturbed quantities as dependent variables, and which contains the applied pressure as a parameter. In all three cases mentioned above it turns out that there are bifurcations, just as in the Euler column theory and the theory of buckling of plates, which manifest themselves through the fact that the homogeneous problems have nontrivial solutions for specific values of the load parameter. These critical loads, and corresponding buckling modes, turn out to be the same as those furnished by the Euler theory, the Föppl-von Kármán theory, and the theory of thin shells, in case the thickness of these solids is made small-thus furnishing some rather strong support for the validity of these approximate theories.

In each of these cases also it turns out that there is an infinite spectrum of critical values and corresponding modes of buckling. The spectrum is a discrete point spectrum, as in the classical eigenvalue problems. However, these linear homogeneous problems have some unusual features in which they differ from the classical eigenvalue problems. For example, while there is an infinite spectrum of buckling modes and corresponding buckling pressures, the latter have for all cases of thick solids examined so far a limit point which is finite;

${ }^{5}$ E. W. Wilkes [44] also solved (prior to Lubkin) a stability problem for a thick solid in the fashion described here. He applies a general perturbation theory due to Green, Rivlin, and Shield [11] to the problem of stability of a circular tube under end thrust, but under the assumption of material which is incompressible. Green and Spencer [12] deal similarly with the cylinder under torsion and lengthwise stretching. Sensenig [36] has solved the buckling problem for the thick circular plate under compression applied to the cylindrical boundary - that is, to the thick plate version of the problem of the circular plate treated by Friedrichs and the speaker. 
only in the limit case of very small thickness do the critical buckling pressures become infinite (as in the Euler column theory, for example). Also, in the case of the buckling of the rectangular solid in two dimensions the bifurcation problem can be formulated in terms of a single function which is a solution of the biharmonic equation, so that the load parameter occurs only in the boundary conditions, and not all in the differential equation. Thus while one obtains an infinite spectrum of "eigenfunctions" it is not clear-in fact, it is perhaps doubtful-that they share the property of the eigenfunctions of classical type, i.e. that the latter form a complete set of functions in terms of which more or less arbitrary functions can be developed.

It would be quite interesting to pursue these problems concerning the buckling of thick-walled solids further. So far, nothing more than the critical loads and corresponding buckling modes has been discussed. Since the relevant problems are linear and homogeneous, it is obvious that the amplitude of the buckling modes is determined only within an arbitrary multiplying factor, and consequently this theory does not make it possible to calculate stresses after buckling has occurred. To do that, it is necessary to pursue the solution into the nonlinear range; in fact something along the lines of what was described above for the case of buckling of the circular plate is necessary. One method of doing so is to carry out the perturbation scheme to higher order, which leads, in particular, to the determination of the amplitudes of the first order variations. This has been done by Grossman [14] for the case of buckling in two dimensions of the rectangular solid; the resulting formulas are, however, very cumbersome and not at all easy to interpret. We had therefore thought that it would be more reasonable to attack the full nonlinear problem for this case of two-dimensional buckling of the rectangular solid numerically, using finite differences, on a digital computer. Although the problem can be formulated in terms of two functions which satisfy a pair of second order elliptic differential equations, and although the problem has only two independent variables, we have not so far found a numerical scheme which delivers a correct numerical solution-illustrating once more the fact noted earlier that something more than fast computers is needed to obtain numerical solutions of nonlinear problems in mathematical physics. It would be quite interesting to have numerical solutions of the problem under discussion here for a range of thickness-length ratios of the solid, since that would make it possible for the first time to decide theoretically whether a given column is so thick that it would fail by crushing under end compression, or so slender that it would fail by becoming unstable and sub- 
sequently be overstressed in bending. For the intermediate range between very thick and very thin columns there is, in other words, no rational design theory in existence at present.

6. Curved elastic surfaces and shells. My lecture has taken a somewhat crooked course, since I began with problems about thinwalled bodies, then went over to thick-walled bodies, and now I begin this portion of my lecture with a different extreme case, namely that of an elastic surface in which the thickness is zero. The theory of elastic surfaces deserves more attention than it has had. It is true that the so-called membrane theory of shells-which is much used in engineering-really belongs in this category of problems, but this theory is of a very special character, as we shall see.

Various problems concerning deformable surfaces have been studied very much in differential geometry and analysis, although they are as a rule not thought of in terms of the theory of elasticity. For example, there is a vast literature devoted to isometric mappings of surfaces immersed in three-dimensional space. It is, of course, intuitively obvious that surfaces in three-dimensional space will admit, in general, isometric mappings which are not necessarily rigid motions, if the boundaries are not subjected to too many constraints, and this means that such bodies admit nontrivial deformations for which the stresses and strains are zero. On the other hand, there is the well-known classical theorem that a closed convex surface in three-dimensional space has no isometric deformations except rigid motions. (Of course, there is involved here a "boundary" condition, i.e. regularity of the deformations over the entire closed surface.) Or, put in a different way, a closed convex elastic surface, if subjected to a deformation that is not a congruence, must of necessity be strained. Thus the theory of elastic surfaces will in general differ greatly from that for solids with inner points in three-dimensional space, since non-trivial deformations are possible without causing stresses and strains. The purely kinematic theory of such isometric deformations has been supplemented by a treatment of the equilibrium states possible in such cases by Beltrami [1], and by Pailloux [30] when the surfaces are subjected to given forces.

Another classical example of an elastic surface is the minimal surface. This surface is commonly characterized as the surface for which the area is stationary when it is subjected to arbitrary variations. From the point of view of elasticity the minimal surface can be characterized by a very simple property. The characterization is that the surface should not be capable of withstanding shear stresses or, put 
differently, that the stress system at every point in the surface is one of constant tension for all directions through a given point, and, in addition, that the external body forces are zero. It is then seen easily that the mean curvature of such a surface is zero, and that, as is well known, is the local necessary condition which results from the variational problem.

In connection with minimal surfaces a curious situation arises which is of interest in view of our earlier preoccupations. It is the following. Suppose one considers a soap film stretched between two parallel circles in such a way as to create a minimal surface in the form of a surface of revolution. It is well known that this minimal surface is generated by a catenary. Such an elastic surface is everywhere under tension, and as the end circles are pulled further apart, the tension increases. Earlier, much has been said about the instability of elastic solids; these instabilities in all cases were created because of the fact that high compressive stresses were developed. I venture to say that if one asked people in the field whether an elastic solid could ever become unstable with increases in a certain load such that the increases are coupled with always increasing tensile stresses everywhere, that they would be inclined to declare such a thing impossible. However, the case just cited of the minimal surface is a counterexample: it is well known that the soap film, if it is stretched too much, eventually becomes unstable and snaps over into two films covering the two end circles. On the other hand, at the moment of instability the film has the form generated by a smooth catenary, the stresses are not large (they are in fact everywhere constant), and there seems to be no obvious reason from the point of view of mechanics which would account for the loss of stability. The fact is that the type of instability exhibited in this case is of a different character from that in all previous cases discussed in this lecture. The transition from an unstable equilibrium position to a stable one does not take place in a continuous fashion; rather, there is a discontinuous jump from one to the other which could be followed only by treating the problem dynamically. Actually, as is well known, the boundary value problem arising from this geometrical question concerning minimal surfaces of revolution simply has no solution at all when the end circles are pulled too far apart. The dynamical aspect of this problem might well be an interesting object for study.

The basic nonlinear theory of elasticity outlined in $\S \S 2$ and 3 above lends itself well to a treatment of elastic surfaces. At the same time it is possible, following Hamel [15], to give an approximate theory of 
thin curved shells in a very efficient and direct way. ${ }^{6} \mathrm{~A}$ thin curved shell is defined by starting with a regular surface in the sense of differential geometry which is defined by a vector

$$
\boldsymbol{X}=\boldsymbol{X}\left(u_{1}, u_{2}\right)=\left(x_{1}, x_{2}, x_{3}\right)
$$

in terms of Gaussian parameters $\left(u_{1}, u_{2}\right)$ which range over a plane domain $D$. To such a surface we adjoin all points on the normals to the surface which extend on both sides of it to a distance $h / 2$ to obtain a curved shell. In other words, $h$ is the small thickness of the thin shell and $\boldsymbol{X}$ defines what is called its middle surface. The only hypothesis which Hamel makes is that the normals to the middle surface map into normals to the middle surface in its deformed position which are straight lines isometric to their pre-images. If we call $X_{3}\left(u_{1}, u_{2}\right)$ the unit normal to the middle surface, the undeformed position of the body is given by

$$
R=X\left(u_{1}, u_{2}\right)+n X_{3}\left(u_{1}, u_{2}\right), \quad-\frac{h}{2} \leqq n \leqq \frac{h}{2},
$$

and for $h$ small enough it is well known that this furnishes a regular curvilinear coordinate system $\left(u_{1}, u_{2}, n\right)$ in the body. In the deformed position we have

$$
\bar{R}=\bar{X}\left(u_{1}, u_{2}\right)+n \bar{X}_{3}\left(u_{1}, u_{2}\right) .
$$

Here, $\bar{X}$ represents the deformed position of the original middle surface $\boldsymbol{X}$, and $\bar{X}_{3}$ is the unit normal of $\bar{X}$; the fundamental hypothesis clearly receives its appropriate formulation in this relation. All writers on the theory of thin elastic shells make this or an equivalent hypothesis.

We can now determine the line element $d s$ of our solid and its image $d \bar{s}$ in terms of the coefficients of the line elements of $X$ and $\bar{X}$ and of the coefficients $L_{i k}$ and $\bar{L}_{i k}$ of their second fundamental forms, which measure curvatures. In fact, we have

$$
d s^{2}=d R \cdot d R=\left(d \boldsymbol{X}+n d \boldsymbol{X}_{3}+\boldsymbol{X}_{3} d n\right)^{2},
$$

and

${ }^{6}$ The problem of deformation of thin curved shells is treated here only incidentally, so that a discussion and analysis of the literature in the field-which is very extensive - will not be attempted. A particular form of the theory, with bibliographical references to earlier literature, is given by Reissner [32]. A recent and different derivation of the theory is given by Koiter [23]; this form of the theory seeks to reduce the simplifying assumptions to a minimum by operating with an appropriate strain energy density function, though not in the same way as in that of Hamel. 


$$
d \bar{s}^{2}=\left(d \overline{\mathbf{X}}+n d \overline{\mathbf{X}}_{3}+\overline{\boldsymbol{X}}_{3} d n\right)^{2} .
$$

The square of the parenthesis on the right-hand side means, of course, that the scalar product is to be taken. We are now in a position to define the strain matrix $\eta$ in a manner analogous to that which led to either (2.4) or $(2.4)^{\prime}$, and can then form its invariants $[\eta]^{2}$ and $\left[\eta^{2}\right]$ which in turn fix the strain energy, once an energy density function $\mathcal{U}$ is chosen, in terms of $\boldsymbol{X}$ and $\bar{X}$, or, rather, in terms of certain very complicated combinations of their first and second derivatives with respect to $u_{1}$ and $u_{2}$. Since the equilibrium problem alone is to be treated here, it follows that the conditions of equilibrium and the boundary conditions (which include the stress-strain relations, as was remarked earlier) result as a consequence of the variational problem

$$
\delta\left\{\int_{-h / 2}^{h / 2} \iint_{D} U\left(g_{11} g_{22}-g_{12}^{2}\right)^{1 / 2} d u_{1} d u_{2} d n+V\right\}=0,
$$

in terms of $V$, the potential energy of the external forces. In the integrand $n$ occurs in an explicit fashion (that is a basic advantageous fact about this method of deriving the approximate theory), and hence can be integrated out. Since $U$ contains second derivatives of all three displacement components $\bar{x}_{i}\left(u_{1}, u_{2}\right)$ of the middle surface-they arise from terms involving $d \bar{X}_{3}$-it follows that the differential equations will be of fourth order, as one expects.

The theory of deformation of thin shells which results from the assumptions leading to (6.3) is peculiar in the sense that the bending stiffness is, as it were, attached to the middle surface. Nevertheless, the natural boundary conditions which result from (6.6) at a free edge, for example, are correctly interpreted as meaning that the applied bending moment and the resultant transverse shear force both vanish. (These are both average quantities with respect to the thickness of the shell.)

I would say that Hamel has dissected the theory of bending of thin shells in such a fashion that its basic and most important hypothesis is singled out, and thus has clarified it quite materially. Indeed, it is easily seen that the theory resulting from (6.6) is quite sufficient, without any additional hypotheses, to solve problems about curved shells subjected to given forces as long as the boundary conditions are given geometrically in terms of displacements (a clamped boundary, for example), or if the boundary is free of transverse forces or if forces having moments about the tangent to the middle surface, and with results identical with those of other authors who work with a 
more complicated basic theory. The theory of Hamel is also very general since it involves no assumptions about the magnitude of the deformations nor any restrictive hypotheses about the stress-strain relations. The theory of Föppl-von Kármán discussed earlier can, for example, be deduced from it (as Hamel [15] does), by making a number of additional restrictive assumptions.

After this digression about the theory of bending of thin shells I return to questions concerning elastic surfaces without bending stiffness. The appropriate mathematical theory for such problems is derived as a special case from (6.6) simply by setting $n=0$, i.e. by taking the thickness of the shell to be zero. If we call $g_{\alpha \beta}$ and $\bar{g}_{\alpha \beta}$ the coeffcients of the line elements of $X$ and $\bar{X}$, we have for them and for the relative elongations $\epsilon=(d \bar{s}-d s) / d s$, the relations

$$
\begin{aligned}
& g_{\alpha \beta}=\frac{\partial x_{j}}{\partial u_{\alpha}} \frac{\partial x_{j}}{\partial u_{\beta}}=x_{j, \alpha} x_{j, \beta}, \\
& \bar{g}_{\alpha \beta}=\frac{\partial \bar{x}_{j}}{\partial u_{\alpha}} \frac{\partial \bar{x}_{j}}{\partial u_{\beta}}=\bar{x}_{j, \alpha} \bar{x}_{j, \beta},
\end{aligned}
$$

with

$$
x_{j, \alpha}=\frac{\partial x_{j}}{\partial u_{\alpha}}, \quad \bar{x}_{j, \alpha}=\frac{\partial \bar{x}_{j}}{\partial u_{\alpha}},
$$

and

$$
\lambda=(1+\epsilon)^{2}=\frac{\bar{g}_{\alpha \beta} d u_{\alpha} d u_{\beta}}{g_{\alpha \beta} d u_{\alpha} d u_{\beta}},
$$

in which the Greek indices range over 1 and 2, while Latin indices continue to range over $1,2,3$. The functions $x_{i}\left(u_{1}, u_{2}\right)$ and $\bar{x}_{i}\left(u_{1}, u_{2}\right)$ are, of course, the components of the vectors $\bar{X}$ and $\bar{X}$. The principal extensions $\epsilon_{i}$ correspond to $\lambda_{i}=\left(1+\epsilon_{i}\right)^{2}$ with $\lambda_{i}$ a root of

$$
\left|\begin{array}{ll}
\bar{g}_{11}-\lambda g_{11} & \bar{g}_{12}-\lambda g_{12} \\
\bar{g}_{22}-\lambda g_{21} & \bar{g}_{22}-\lambda g_{22}
\end{array}\right|=0 .
$$

It is therefore clear that the following are invariants in terms of which the strain energy density can properly be defined:

$$
\begin{aligned}
& r=\frac{g_{11} \bar{g}_{22}-2 g_{12} \bar{g}_{12}+g_{22} \bar{g}_{11}}{g_{11} g_{22}-g_{12}^{2}}, \\
& s=\frac{\bar{g}_{11} \bar{g}_{22}-\bar{g}_{12}^{2}}{g_{11} g_{22}-g_{12}^{2}} .
\end{aligned}
$$


Thus $U$ in (6.6) is a certain function $U=U(r, s)$. The variational equations resulting from (6.6) can then be put in the form:

$$
\begin{aligned}
& \frac{\partial}{\partial u_{\alpha}}\left(\frac{\partial \mathcal{U}}{\partial \bar{x}_{i, \alpha}}\left(g_{11} g_{22}-g_{12}^{2}\right)^{1 / 2}\right)+F_{i}\left(\bar{g}_{11} \bar{g}_{22}-\bar{g}_{12}^{2}\right)^{1 / 2}=0, \\
& i=1,2,3 .
\end{aligned}
$$

Boundary conditions, and with them the stress-strain relations, would also be obtained. In the set (6.10) of three equations for the functions $\bar{x}_{i}$ the quantities $F_{i}$ represent the components of the external forces per unit area in the deformed position. From (6.7), (6.8), and (6.9) it is clear that these differential equations are all of second order in the components $\bar{x}_{i}$ of $\bar{X}$ as dependent quantities. This is what one might reasonably expect, since it would seem physically appropriate to prescribe values for the three displacement components at the boundary (for example, that all of them should vanish if the boundary of the surface is supposed fixed), and three second order partial differential equations for the quantities $\bar{x}_{i}$ are therefore needed. But then the differential equations should also be of elliptic type, and that gives rise to a number of observations of interest from more than one point of view.

It might seem odd that the remarks just made should be necessary since one expects that any reasonably formulated equilibrium problem in continuum mechanics should lead to a system of differential equations of elliptic type, and that the condition of a fixed boundary would then be a legitimate boundary condition for them. However, the great bulk of the literature about elastic surfaces concerns itself with a basic theory in which neither of these expectations may be realized. This theory, to which a passing reference was made earlier, is called the membrane theory of shells, and it has considerable practical value and importance for the design of structures of quite varied character and use (see, for example, the books by Flügge [8] and Novozhilov [29]).

The theory presented in these books has a very simple basis. One assumes that the stresses throughout the thickness of the shell are the same as in the middle surface, and that the deformed middle surface represented by $\bar{X}$ differs by an infinitesimal of first order from the original undeformed surface given by $X$, so that it becomes legitimate as a first approximation to write down equilibrium conditions in terms of stress, and, what is more, write down these conditions in the undeformed position, which is of course known. Thus there would be three linear equations for the three unknown stresses, and these would correspond to the three equations (6.10) of the general 
nonlinear theory. This all sounds quite reasonable, and in fact for many important practical problems it is reasonable.

Nevertheless, the theory has queer features, and it is not always sufficient, even in practice. To begin with, the mechanical system postulated is a statically determinate system, since the three stress components are determined from the applied loads from the three conditions of equilibrium alone and without any necessity to consider deformations and strains. Afterwards, upon introducing the customary linear relations between stress and strain it is possible to determine the strains, and from them the displacements. In general one is in no position to prescribe the displacements arbitrarily at the boundary; in particular it is not possible in general to prescribe a fixed boundary. Also, it turns out that the system of equations for the stresses can be reduced to a single second order partial differential equation for one of the stresses as a function of the surface parameters $\left(u_{1}, u_{2}\right)$, and this linear equation is elliptic or hyperbolic depending upon whether the original surface has positive or negative Gaussian curvature. (If the Gaussian curvature is zero, so that the original middle surface is a developable, the differential equation is parabolic or degenerate.) In practice, the structures dealt with (roofs of buildings, etc.) are usually at least locally convex (i.e. the Gauss curvature is positive), so that the engineers need not try to solve boundary value problems for hyperbolic equations. The disagreeable feature that fixed boundaries are not allowed is commonly dealt with by invoking bending stiffness so that a system of differential equations of higher order results, and that, even in the linear theory, permits the imposition of conditions corresponding to a fixed boundary. However, I myself once had occasion to deal with a problem in which the shell was so thin that bending stiffness was effectively nonexistent, but still it was quite necessary to have a fixed boundary. ${ }^{7}$ In such a case, resort must be had to a nonlinear theory-for example, that embodied in (6.10); actually, the problem was solved (cf. [5]) by using a theory which is a simplified version of this general nonlinear theory.

The last remarks raise an interesting question, i.e. what is the relation between the classical linear membrane theory of shells and the

7 The problem was that of a so-called air-supported roof. During the War it was proposed to erect buildings in circular form with a diameter of several hundred feet roofed over by a thin steel sheet perhaps $1 / 8^{\prime \prime}$ in thickness (and hence clearly so thin that the bending stiffness would be negligible), and blown up to provide headroom, without interior supports to obstruct the storage space, by excess air pressure. The idea is not as silly as it might sound: only a few ounces per square inch of internal excess air pressure are required to hold such a roof in place and even to strain it up to the yield point for steel. 
theory embodied in equations (6.10), and I wish to analyze it briefly. The basic fact of the matter is that equations (6.10) are elliptic only with respect to solutions for which the principal stresses are everywhere tensions, not compressions. Thus the type of these equations is not fixed by the sign of the Gaussian curvature of the surface in its original position, as is the case in the classical linear membrane theory. On the other hand, the two theories should be identical when the system (6.10) is linearized, which means that, here also, these equilibrium conditions are written down for the surface in its original undeformed position. This would appear to be a contradiction, since this system is in general elliptic provided only that the stresses turn out to be tensions and independent of the Gaussian curvature of the surface. However, the fact is that the system (6.10) degenerates in order when it is linearized so that here also the imposition of boundary conditions corresponding to a fixed edge is not possible. What this means is that the linearized theory of elastic surfaces, unlike that for three-dimensional elastic bodies, is not a regular perturbation theory in which the dependent quantities are developable in power series in a small parameter which converge in the neighborhood of the unstressed and unstrained state; instead, this linear theory of elasticity is an asymptotic theory in such a parameter in which boundary layer effects are present and cannot be ignored if conditions on all three displacement components are imposed at a boundary; hence the underlying nonlinear theory must be invoked in such cases. This point of view was one of the main features of the paper by Bromberg and the speaker [5] cited earlier, but for a simplified version of the theory embodied in equations (6.10) and for the special case of a spherical surface deformed into a surface of revolution, so that the question of ellipticity was not relevant. However, the condition on the sign of the normal stresses turned up in another connection in this case, i.e. with respect to Legendre's necessary condition concerning the second variation of the potential energy: only if the normal stresses are tensions can the equilibrium position correspond to a minimum of the potential energy. Though this phenomenon has not been investigated in the general case embodied in equations (6.10), it seems highly probable that it would hold there also, and thus lead to the conclusion that stable equilibrium positions of an elastic surface are not possible if a compressive stress occurs anywhere-and that coincides with what one would instinctively feel to be correct physically. In the classical linear membrane theory, on the other hand, all equilibrium positions are stable. In effect, the linearization is tantamount in these cases to the imposition of a constraint strong enough to ensure 
stability. In practice, shells are usually loaded in such a way as to be in compression, and hence their positions of equilibrium correspond to unstable states of the system characterized by the underlying general nonlinear theory. However, such shells are commonly made stable by virtue of their bending stiffness, and it is one of the many odd features of the linear membrane theory of shells that it is used successfully in practice even though the design formulas ignore exactly that feature which alone makes the shell stable, i.e. its bending stiffness.

In connection with problems of elastic surfaces centering around (6.10) it is worthwhile to consider their relation to problems about inextensible but perfectly flexible surfaces, and particularly for closed surfaces. A classic problem, already mentioned, was that of the uniqueness within orthogonal transformations of the closed convex surfaces under isometric deformations - a fact proved in a wholly satisfactory way by Herglotz [16] relatively recently. Nothing much is known about the case of nonconvex closed surfaces, nor about closed surfaces which are not homeomorphic with the sphere; these cases are made awkward by the fact that the relevant partial differential equations (of Monge-Ampère type) are not elliptic everywhere. In elasticity, however, the differential equations are in general of elliptic type. Thus the uniqueness theorems for closed surfaces might be more amenable to treatment-even if bending stiffness is also taken into account (when, in fact, the differential equations are certainly elliptic) - so that generalizations of classic problems in differential geometry in the large might be within reach. Also, the limit cases of zero bending stiffness, and of inextensibility of the surface, are degenerate cases in which boundary layer phenomena are to be expected because of the loss of order of the differential equations in the limit: there exists, in other words, a hierarchy of limit problems of this sort. In effect, there is a field for study here which could be quite fruitful from more than one point of view.

7. Conclusion. In my introductory remarks I noted that twenty years have passed since I addressed the Society at its invitation. It struck me that I might therefore by reason of age alone be permitted to make a few remarks of a general character about mathematics in our country, especially with regard to some aspects of it which touch my own personal interest and bias.

It is a strange thing that in this country, which prides itself so much on the practical use it makes of all the scientific knowledge which has been gathered over the centuries, mathematics has been pursued for the last fifty years or more in a pronouncedly abstract manner, 
and that that side of our science in which the relation between mathematics and the physical world plays an important role has been very much neglected. Willard Gibbs was in this respect an exception among mathematicians born in the United States. It should hardly be necessary to add that the accomplishments of mathematicians in this country have been notable indeed-in fact, the obvious high quality of this work and the attraction of good students to those who had carried it out were doubtlessly the main factors at play in fixing the mathematical climate here. There have also been outstanding individuals who deviated from the norm, such as G. D. Birkhoff, for example.

There has been some change in this situation in the last ten or fifteen years in this country, which was brought about largely by mathematicians who came here from Europe in the thirties and early forties. To this day, most of the better-known mathematicians who pursue what is called applied mathematics in this country were European born and trained. This group of Europeans - the people in it are mostly well known to you all-has induced considerable changes; but I observe that the abstract point of view and the neglect, even the contempt, for that kind of mathematics which concerns itself with the world of reality, still represents the prevailing tone in American mathematics. The plain fact is that the leading practitioners of that branch of mathematics in which the interplay with mechanics and physics is a strong motivation are nearly all of the older generation, and there seem to be very few replacements for them in sight. In my view this is not a healthy state-neither for our science itself, nor for the welfare of this country. Furthermore, there are strong forces at work, I observe, which have the tendency to perpetuate this situation by propagating the notion that the strongly abstract approach to mathematics is the suitable way to introduce it to children in the elementary schools. It would seem to me that this attitude ignores human psychology and turns reason upside down: it ignores the historical fact that the mode of progress in mathematics has always consisted in formulating the appropriate and truly valuable abstractions on the basis of prolonged experience of a very concrete character, and the accompanying highly plausible inference that that is also the way most people's minds work.

It should not be thought that I and other colleagues who share my attitude feel ourselves in opposition to those mathematicians who chose to pursue their work in as abstract a fashion as they find suitable and rewarding. Thus we have no particular quarrel with Bourbaki - that constitutes serious mathematical work which needs no com- 
ment from us; it is rather with the petit Bourbaki that we have our quarrel. Our point of view is that it is vital for the health of our science that the contact with the physical world should be preserved and cultivated, not merely because of the obvious practical achievements which inevitably result from such work, but because the whole history of mathematics shows that such preoccupations have a stabilizing, vitalizing, and fruitful effect on our science.

For many years I have found it a pleasure to read poetry in several languages, and since there is one poem of Goethe which reinforces the view just expressed (though not without a certain element of ambiguity), I take the liberty of closing my lecture by quoting two stanzas from it:

\author{
Denn mit Göttern \\ Soll sich nicht messen \\ Irgendein Mensch. \\ Hebt er sich aufwärts \\ Und berührt \\ Mit dem Scheitel die Sterne, \\ Nirgends haften dann \\ Die unsichern Sohlen, \\ Und mit ihm spielen \\ Wolken und Winde. \\ Steht er mit festen, \\ Markigen Knochen \\ Auf der wohlgegründeten \\ Dauernden Erde, \\ Reicht er nicht auf, \\ Nur mit der Eiche \\ Oder der Rebe \\ Sich zu vergleichen.
}

\title{
BIBLIOGRAPHY
}

1. E. Beltrami, Sull' equilibrio delle superficie flessibili ed inestensibile, Mem. Acc. Sci. di Bologna, 1881-1882.

2. M. A. Biot, Nonlinear theory of elasticity and the linearized case for a body under initial stress, Phil. Mag. 27 (1939).

3. - Theory of elasticity with large displacements and rotations, Proc. 5th Int. Congress Appl. Mech., 1958.

4. E. Bromberg, Nonlinear bending of a circular plate under normal pressure, Comm. Pure Appl. Math. 9 (1956).

5. E. Bromberg and J. J. Stoker, Nonlinear theory of curved elastic sheets, Quart. Appl. Math. 3 (1945).

6. W. Z. Chien, The intrinsic theory of thin shells and plates. I, II, III, Quart. Appl. Math. 1 (1944), 297-327; 2 (1944), 43-59; 2 (1944), 120-135. 
7. Paul Fife, An elastic plate problem with boundary layer: asymptotic treatment and a priori pointwise estimates, Thesis, New York Univ., New York, 1959.

8. W. Flügge, Statik und Dynamik der Schlalen, Edwards Brothers, Ann Arbor, Mich., 1943.

9. K. O. Friedrichs, Asymptotic phenomena in mathematical physics, 28th Josiah Willard Gibbs lecture, Pittsburgh, 1954, Bull. Amer. Math. Soc. 61 (1955), 485-504.

10. K. O. Friedrichs and J. J. Stoker, The nonlinear boundary value problem of the buckled plate, Amer. J. Math. 63 (1941).

11. A. E. Green, R. S. Rivlin and R. T. Shield, General theory of small elastic deformations superposed on finite elastic deformations, Proc. Roy. Soc. London. Ser. A 211 (1952), 128-154.

12. A. E. Green and A. J. M. Spencer, The stability of a circular cylinder under finite extension and torsion, J. Math. Phys. 37 (1959), 316-338.

13. A. E. Green and W. Zerna, Theoretical elasticity, Clarendon Press, Oxford, 1954.

14. Norman Grossman, Non-linear problems in elastic stability, Thesis, New York Univ., New York, 1958.

15. G. Hamel, Theoretische Mechanik, Springer, Berlin, 1949.

16. G. Herglotz, Ueber die Starrheit der Eiflaechen, Abh. Math. Sem. Hansischem Univ. 15 (1943), 127-129.

17. F. John, On finite deformations of an elastic isotropic material, New York Univ., Institute of Math. Sciences, IMM-NYU 250, 1958.

18. - Plane strain problems for a perfectly elastic material of harmonic type, Comm. Pure Appl. Math. 13 (1960).

19. - Rotation and strain, Comm. Pure Appl. Math. 14 (1961), 391-413.

20. R. Kappus, Zur Elastizitätstheorie endlicher Verschiebungen, Z. Angew. Math. Mech. 19 (1939), 271, 344.

21. Th. von Kármán, Festigkeitsprobleme im Maschinenbau, Enzykl. Math. Wiss. 4 (1910).

22. W. T. Koiter, On stability of elastic eguilibrium, Thesis, Delft, 1945. (Dutch)

23. - A consistent first approximation in the general theory of thin elastic shells, Proc. I.U.T.A.M. Sym. on the Theory of Thin Elastic Shells, Delft, 1959, p. 12.

24. K. Kreutzer, Die Stabilität des gedrückten Stabes, Z. Angew. Math. Mech. 12 (1932), 351-368.

25. A. E. H. Love, Theory of elasticity, 4th Ed., Dover, New York, 1944.

26. S. Lubkin, Determination of buckling criteria by minimization of total energy, New York Univ., Institute of Math. Sciences, Report No. 241, 1957.

27. F. D. Murnaghan, Finite deformations on an elastic solid, Amer. J. Math. 59 (1937); Wiley, New York, 1951.

28. V. V. Novozhilov, Foundations of the non-linear theory of elasticity, Graylock Press, Rochester, N. Y., 1939.

29. - The theory of thin shells, English translation from the Russian, Noordhoff, Groningen, 1959.

30. H. Pailloux, Contribution a l'étude des systèmes déformables, Ann. de Toulouse 5 (1937).

31. L. Prandtl, The mechanics of viscous fluids, Aerodynamic Theory, Vol. 3, Springer, Berlin, 1935.

32. E. Reissner, $A$ new derivation of the equations for the deformation of elastic shells, Amer. J. Math. 63 (1941). 
33. - On a variational theorem for finite elastic deformations, J. Math. Phys. 32 (1953), 129-135.

34. - The edge effect in symmetric bending of shallow shells of revolution, Comm. Pure Appl. Math. 12 (1959), 385-398.

35. R. S. Rivlin, Some topics in finite elasticity, Brown Univ., Div. Appl. Math., Tech. Rep. No. 43, 1958.

36. Chester B. Sensenig, The buckling of a thick circular plate using a non-linear theory, IMM-NYU 262, December 1959.

37. J. J. Stoker, Bending and buckling of elastic plates, Lecture Notes, New York Univ., 1941.

38. S. Timoshenko, Theory of elastic stability, McGraw-Hill, New York, 1936.

39. J. L. Synge and W. Z. Chien, The intrinsic theory of elastic shells and plates. Theodore von Kármán Anniversary Volume, pp. 103-120, California Institute of Technology, Pasadena, Calif., 1941.

40. E. Trefftz, Ueber die Ableitung der Stabilitaetskriterien des elastischen Gleichgewichts aus der Elastizitaets-theorie endlicher Deformationen, Proc. of the 3rd International Congress for Technical Mechanics, Vol. 3, Stockholm, 1930, p. 44.

41. - Zur Theorie der Stabilitaet des elastischen Gleichgewichts, Z. Angew. Math. Mech. 13 (1933), 160-165.

42. C. Truesdell, The mechanical foundations of elasticity and fluid dynamics, J. Rational Mech. Anal. 1 (1952), 125-300.

43. - The rational mechanics of materials-past, present, future, Appl. Mech. Rev. 12 (1959), 75-80.

44. E. W. Wilkes, On the stability of a circular tube under end thrust, Quart. J. Mech. Appl. Math. 8 (1955), 88-100.

45. M. Yanowitch, Nonlinear buckling of a circular plate under normal pressure, Comm. Pure Appl. Math. 9 (1956), 661-672.

New York UnIversity 